(ECM). Hepatic progenitor cell (HPC) activation occurs in the context of severe liver injury. ECM stiffness has been shown to direct differentiation in mesenchymal stem cells. However, the effect of mechanical factors, such as ECM stiffness on HPC responses is poorly characterised. We examined the effect of ECM stiffness on HPC proliferation and differentiation.

Methods Experiments were undertaken using a murine HPC line (BMOL) and primary murine HPCs. Cell culture experiments were performed using a system of laminin-coated polyacrylamide (PA) gel supports of variable stiffness. The stiffness of the PA supports (expressed as shear modulus) was altered across a physiological range $(1-12 \mathrm{kPa})$ corresponding to values encountered in normal and fibrotic livers.

Results Increasing matrix stiffness is associated with enhanced cell spreading. BMOL cells cultured on stiff $(12 \mathrm{kPa})$ supports develop prominent actin stress fibres. The projected surface area (mean\pm SEM) of BMOL cells culture on soft $(1 \mathrm{kPa})$ supports was $378 \pm 21 \mu \mathrm{m}^{2}$ compared to $687 \pm 47 \mu^{2}$ for BMOL cells cultured on stiff $(12 \mathrm{kPa})$ supports $(\mathrm{p}<0.001)$. Cell proliferation (Ki67 positivity) increased as a function of increasing matrix stiffness. The proliferative index (PI) of BMOL cells cultured on $2.5 \mathrm{kPa}$ and $12 \mathrm{kPa}$ supports was 7.1-fold $(p<0.01)$ and 11.8-fold higher $(p<0.001)$, respectively, than cells cultured on $1 \mathrm{kPa}$ supports. Similarly, in experiments with primary cells, the PI of murine HPCs was 1.7-fold higher $(p<0.05)$ when cells were cultured on stiff $(12 \mathrm{kPa})$ vs soft $(1 \mathrm{kPa})$ supports. Quantitative PCR revealed that BMOL cells cultured on soft $(1 \mathrm{kPa})$ supports up-regulate hepatocyte markers, including; albumin (1.5-fold, $\mathrm{p}<0.01)$ and CYP7A1 (1.6-fold, $\mathrm{p}<0.01)$, and down-regulate the HPC/biliary marker cytokeratin-19 (0.6-fold, $\mathrm{p}<0.01)$, relative to cells on stiff $(12 \mathrm{kPa})$ supports. There was no significant change in expression of the biliary epithelial cell markers aquaporin- 1 and $\gamma$-glutamyl-transferase.

Conclusion Physiological changes in ECM stiffness lead to alterations in HPC morphology, proliferation and differentiation. Increased ECM stiffness (as would be encountered in an injured or fibrotic liver) promotes HPC proliferation and expression of the $\mathrm{HPC} /$ biliary marker cytokeratin-19. In contrast, a low-stiffness environment is associated with a reduction in cell proliferation and up-regulation of hepatocyte-specific markers. These results suggest that mechanical factors, such as ECM stiffness might regulate HPC responses following liver injury.

Competing interests None declared.

\section{OC-158 ENDOGENOUS PRODUCTION OF ANTIBIOTICS BY MESENCHYMAL STEM CELLS AND THE POTENTIAL VALUE IN CROHN'S FISTULA HEALING}

doi:10.1136/gutjnl-2012-302514a.158

${ }^{1} \mathrm{~L}$ Meran, ${ }^{*} \mathrm{M}$ Garavaglia, ${ }^{2} \mathrm{~S}$ Heeb, R Bayston, B Richards, ${ }^{3} \mathrm{~W}$ Ashraf, ${ }^{4} \mathrm{~F}$ Rose, ${ }^{4} \mathrm{~K}$ Shakesheff, ${ }^{1} \mathrm{C}$ Hawkey. ${ }^{1}$ Nottingham Digestive Diseases Centre, Nottingham University Hospitals, Nottingham, UK; ${ }^{2}$ Centre for Biomolecular Sciences, Nottingham, UK; ${ }^{3}$ Biomaterials Related Infection Group, University of Nottingham, Nottingham, UK; ${ }^{4}$ Tissue Engineering, STEM group, Centre for Biomolecular Sciences, Nottingham, UK

Introduction Potent effects of human mesenchymal stem cells (MSCs) on immune responses have been demonstrated in vitro studies and animal models, encouraging further investigations into their clinical value. Studies of MSCs in fistula healing have emphasised the immunosuppressive mechanisms of action however, there has been little focus on their antibacterial activity in this setting. We report here on the antibacterial effects of MSCs against Adherent Invasive Escherichia coli (AIEC), reference strain LF82.

Methods Cultured human bone marrow derived MSCs were plated at a density of $5 \times 10^{4}$ cells per square centimetre in 24 well plates and allowed to adhere overnight. Confluent MSCs were preincubated for 1-day with fresh culture medium alone or medium supplemented with $1 \mathrm{ng} / \mathrm{ml}$ TNFa. Cells of passage numbers 4-9 were co-cultured with a calculated 300 Colony Forming Units of AIEC strain LF82 and incubated for $6 \mathrm{~h}$ in a humidified $\mathrm{CO}_{2}$ incubator. Aliquots of the infected medium were taken from each well and plated on LB-agar plates and colonies were counted after overnight incubation. Comparisons were made with aliquots from inoculated wells containing cells that were pre-stimulated with TNF $\alpha$, in addition to wells that did not contain mesenchymal stem cells. Data analyses were performed using the SPSS statistical package (V.19.0).

Results Each of the wells under test conditions was inoculated with $379 \pm 98 \mathrm{CFU}$ at the start of the co-culture time period. In the absence of mesenchymal stem cells, the number of LF82 CFUs in mesenchymal stem cell culture medium had risen to a mean $( \pm S D)$ of $1780 \pm 319$ [n=3], after $6 \mathrm{~h}$. In the presence of passage- 4 MSCs, the number of LF82 CFU was significantly reduced by $81.2 \%$, to $335 \pm 61[n=3] \quad(p<0.05)$. When MSCs were prestimulated with TNF, there was a further reduction of CFU levels to $15 \pm 6[n=3]$. When cells of higher of passage numbers were used, the effects both in the presence and absence of TNF prestimulation diminished such that by passage- 8 , there was no significant inhibition of bacterial growth.

Conclusion The antibacterial properties of MSCs alongside their tissue regenerative potential make them ideal candidates for use in therapeutic strategies for fistula healing. This study demonstrates that in a proinflammatory environment, MSCs enhance bacterial clearance of AIEC. These findings encourage further evaluation of the antimicrobial effector function of MSCs against other strains suspected to participate in the pathogenesis of Crohn's disease.

Competing interests None declared.

\section{BSG information group symposium \& free papers: "Social media and apps: new oppor- tunities, new risks" \\ OC-159 CONTENT ANALYSIS OF ILLNESS BLOGS POSTED ONLINE BY PATIENTS WITH IBD}

doi:10.1136/gutjnl-2012-302514a.159

N Tshuma, ${ }^{*}$ S Aboulela, F Chowdhury, L Macken, N Kamperidis, J Goodhand, J Lindsay. Department of Gastroenterology, Barts and the London NHS Trust, London, UK

Introduction Illness blogs are online accounts of the course of a disease: they are unsolicited first person narratives, that are publicly accessible allowing author-reader interactivity. Expressive writing improves quality of life and scores in patients with irritable bowel syndrome. We sought to compare the accessibility and contents of illness blogs written by patients with ulcerative colitis (UC) and Crohn's disease (CD): hypothesising that psychological distress, reportedly more common in IBD than the general population, and active disease would be the key reasons for a post.

Methods Using the search terms "Crohn's* and Ulcerative colitis* with Blog" and the internet search engine Google, we identified 12 consecutive UC and Crohn's disease illness narratives. All posts written in the preceding year were included. We then undertook a structured quantitative analysis; including an assessment of the readability of posts using the Flesch reading ease (FRE) score and author-reader interactivity. Semi-qualitative analyses of excerpts of the contents of each post were undertaken using Dedoose software to identify narrative themes: wherever possible, for each post, disease activity was recorded.

Results 1297 and 1009 excerpts were made from 642 and 499 posts from the illness blogs written by patients with UC and CD, respectively. There were no differences in the mean [SD] number of 